Research Article

\title{
A Novel MIMO Array with Reduced Mutual Coupling and Increased Degrees of Freedom
}

\author{
Mingxin Liu (D), ${ }^{1}$ Lin Zou $\mathbb{D}^{2},{ }^{2}$ Haohao Ren, ${ }^{2}$ Xuelian Yu, ${ }^{2}$ Yun Zhou, ${ }^{2}$ and Xuegang Wang ${ }^{2}$ \\ ${ }^{1}$ School of General Aviation, Chengdu Aeronautic Polytechnic, Chengdu 610100, China \\ ${ }^{2}$ School of Information and Communication Engineering, University of Electronic Science and Technology of China, \\ Chengdu 611731, China \\ Correspondence should be addressed to Mingxin Liu; lmx0951@163.com
}

Received 17 July 2020; Revised 27 January 2021; Accepted 3 February 2021; Published 15 February 2021

Academic Editor: Maria Angela Butturi

Copyright (c) 2021 Mingxin Liu et al. This is an open access article distributed under the Creative Commons Attribution License, which permits unrestricted use, distribution, and reproduction in any medium, provided the original work is properly cited.

In this paper, we consider the problem of array design for Multiple-Input Multiple-Output (MIMO) array under the condition of fixed number of physical sensors and mutual coupling. A novel MIMO array based on the second-order super nested transmit and receive arrays is proposed by using the difference coarray. It can obtain the closed form expressions for the physical sensor locations and the degrees of freedom (DOF) from any given number physical sensors. The proposed array structure can significantly enhance DOF and effectively decrease unknown mutual coupling effect. The effectiveness and superiority of the proposed MIMO array structure are verified from the number of DOF and MUSIC spectra by numerical simulations.

\section{Introduction}

Multiple-Input Multiple-Output (MIMO) radar transmits orthogonal or incoherent multiple probing signals and receives echo signals by utilizing matched filter banks, which provides more degrees of freedom (DOF) in the design of radar systems and signal processing algorithms [1-4]. In view of the configurations of the transmit and receive arrays, the MIMO radar systems can be differentiated between distributed MIMO radar [5] and colocated MIMO radar $[6,7]$. By exploiting waveform diversity, the colocated MIMO radar can improve the target detection, estimation, and parameter identification capability.

The problem of array structure for the colocated MIMO radar has received considerable attention recently. Currently, most open literatures mainly have focused on research of the sparse arrays with closed expressions such as nested arrays [8-10], coprime arrays [11-17], and minimum redundancy arrays $[18,19]$. They can offer a longer virtual aperture compared to uniform linear arrays (ULA) by utilizing second-order statistics of the received data. Hence, the number of detectable sources is bigger than the number of sensors. The minimum redundancy MIMO array was proposed in [18] based on difference basis to obtain a large virtual array aperture with only few sensors. The system realizes the virtual expansion of receive array by difference coarray of the sum coarray (DCSC), thus greatly increases DOF [20]. However, the minimum redundancy MIMO array usually has disadvantages of huge calculating quantity and long computing time. By replacing the minimum redundancy arrays with the coprime arrays, we gain the coprime MIMO array which can reduce the calculate burden and increase the freedom of the array [14]. Sad to say, it cannot use the spatial smoothing algorithm to decompose the correlated signals and then estimate the direction of arrival accurately on account of the holes in its coarray. Similarly, the nested MIMO array can make further efforts to enhance the DOF and produce a hole-free difference coarray, while the nested arrays contain a dense physical ULA by definition to result in higher mutual coupling $[21,22]$.

In this paper, we investigate the problem of increasing virtual aperture for the colocated MIMO radar with considering the unknown coupling and propose a novel MIMO array structure. More specifically, we introduce a secondorder super nested arrays structure into the MIMO radar 
transmit and receive arrays to bring less mutual coupling. Simultaneously, we also establish the necessary condition to maximize the DOF of the corresponding hole-free DCSC. Therefore, the proposed array structure has the following advantages: (1) the closed form sensor locations and freeholes DCSC, (2) higher DOF than coprime arrays and ULA structure in given physical sensors, and (3) less coupling compared to the nested arrays and ULA structure.

The remainder of this paper is organized as follows. We introduce the signal model and mutual coupling and construct the proposed array structure in Section 2. Section 3 gives the comparisons between the proposed array structure and other methods versus DOF and MUSIC spectra. Section 4 concludes this paper.

\section{The Proposed Array Structure}

2.1. Signal Model. The colocated MIMO radar system which possesses $M$ transmit sensors with $\left\{m_{j} d \mid m_{j} \in \mathbb{T}, j=1, \ldots, M\right\}$ and $N$ receive sensors with $\left\{n_{i} d \mid n_{i} \in \mathbb{R}, i=1, \ldots, N\right\}$ transmits the orthogonal waveforms to detect $D$ uncorrelated sources from directions $\theta_{i}(i=1,2, \ldots, D)$ with powers being $\delta_{i}^{2}$. Here, $\mathbb{T}$ and $\mathbb{R}$ denote integer sets, and the minimum distance between sensors $d$ is a half wavelength. Then, the received data after matched filtering can be modeled as

$$
\mathbf{x}(t)=\mathbf{A s}(t)+\mathbf{n}(t),
$$

where the array manifold matrix is

$$
\mathbf{A}=\left[\mathbf{a}_{T}\left(\theta_{1}\right) \otimes \mathbf{a}_{R}\left(\theta_{1}\right), \mathbf{a}_{T}\left(\theta_{2}\right) \otimes \mathbf{a}_{R}\left(\theta_{2}\right), \ldots, \mathbf{a}_{T}\left(\theta_{D}\right) \otimes \mathbf{a}_{R}\left(\theta_{D}\right)\right],
$$

in which

$$
\begin{aligned}
& a_{T}\left(\theta_{i}\right)=\left[e^{\pi j m_{1} \sin \theta_{i}}, e^{\pi j m_{2} \sin \theta_{i}}, \ldots, e^{\pi j m_{M} \sin \theta_{i}}\right]^{T}, \\
& a_{R}\left(\theta_{i}\right)=\left[e^{\pi j n_{1} \sin \theta_{i}}, e^{\pi j n_{2} \sin \theta_{i}}, \ldots, e^{\pi j n_{N} \sin \theta_{i}}\right]^{T},
\end{aligned}
$$

are the transmit and receive steering vectors, respectively, $\otimes$ stands for the Kronecker product, and $\mathbf{s}(t)$ and $\mathbf{n}(t)$ are the input source vector and the Gaussian white noise vector with power being $\sigma_{n}^{2}$, respectively. The covariance matrix can be estimated from $L_{s}$ snapshots as follows:

$$
\mathbf{R}=E\left[\mathbf{x} \mathbf{x}^{H}\right]=\mathbf{A R}_{s} \mathbf{A}^{H}+\sigma_{n}^{2} \mathbf{I} \approx \frac{1}{L_{s}} \sum_{l=1}^{L_{s}} \mathbf{x}(l) \mathbf{x}^{H}(l),
$$

where $\mathbf{R}_{s}=E\left[\mathbf{s s}^{H}\right]=\operatorname{diag}\left\{\left[\delta_{1}^{2}, \delta_{2}^{2}, \ldots, \delta_{D}^{2}\right]^{T}\right\}$ is the covariance matrix of input source and I denotes the identity matrix. According to the nature of MIMO array, the term $\mathbf{a}_{T}\left(\theta_{i}\right) \otimes \mathbf{a}_{R}\left(\theta_{i}\right)$ can be interpreted as the steering vector of a virtual array with $M N$ sensors whose positions are expressed as

$$
\begin{aligned}
& \left\{o_{k} d \mid o_{k} \in \mathbb{O}, \quad k=1, \ldots, M N\right\} \\
& \quad=\left\{m_{j} d+n_{i} d \mid m_{j} \in \mathbb{T}, \quad j=1, \ldots, M ; n_{i} \in \mathbb{R}, i=1, \ldots, N\right\},
\end{aligned}
$$

where the set $\mathbb{O}$ means the sensor positions of virtual array that can be called the sum coarray of the transmit and receive arrays. The difference coarray of the virtual array are given by the set $\mathbb{A}$ defined as

$$
\begin{aligned}
\mathbb{A} & =\left\{o_{k 1} d-o_{k 2} d \mid o_{k 1}, o_{k 2} \in \mathbb{O}, \quad k 1, k 2=1, \ldots, M N\right\} \\
& =\left\{\begin{array}{c}
m_{j 1} d+n_{i 1} d-m_{j 2} d-n_{i 2} d \mid \\
m_{j 1}, m_{j 2} \in \mathbb{T}, \quad j 1, j 2=1, \ldots, M ; n_{i 1}, n_{i 2} \in \mathbb{R}, i 1, i 2=1, \ldots, N
\end{array}\right\} .
\end{aligned}
$$

For a fixed number of sensors, we can gain the transmit and receive arrays' positions to maximize the DOF of the hole-free difference coarray by solving the following optimization problems:

$$
\begin{array}{ll}
\max _{\{\mathbb{\mathbb { T }}\},\{\mathbb{R}\}}, L, \\
\text { s.t. } & |\mathbb{\mathbb { T }}|=M,|\mathbb{R}|=N,\left\{m_{j 1}+n_{i 1}-m_{j 2}-n_{i 2}\right\} \\
& \supset\{-L, \ldots,-1,0,1, \ldots, L\},
\end{array}
$$

where $L$ is the continuous aperture length and $|\bullet|$ represents the cardinality of the set.

2.2. Mutual Coupling. In practical array, the received signal has changed owing to the electromagnetic coupling between the sensors. Here, $\mathbf{C}_{T}$ and $\mathbf{C}_{R}$ represent the mutual coupling matrices of the transmit and receive arrays, respectively.
According to [23], $\mathbf{C}_{T}$ and $\mathbf{C}_{R}$ of the ULA can be formulated by a B-banded mode

$$
\begin{aligned}
& \mathbf{C}_{T}=\text { Toeplitz }\left[c_{0}, c_{1}, \ldots, c_{B}, 0, \ldots, 0\right], \\
& \mathbf{C}_{R}=\operatorname{Toeplitz}\left[c_{0}, c_{1}, \ldots, c_{B}, 0, \ldots, 0\right],
\end{aligned}
$$

where the mutual coupling coefficients $c_{i}(i=0, \ldots, B)$ satisfy $\left|c_{B}\right|<\cdots<\left|c_{1}\right|<\left|c_{0}\right|=1$. In the actual system, we need to give careful consideration to the mutual coupling effect which will distort the steering vectors of the transmitter and receiver.

2.3. The Proposed MIMO Array. In this paper, we assume that the total number of physical sensors of MIMO array is $K=N+M$, whose transmit ( $\mathrm{Tx})$ and receive ( $\mathrm{Rx})$ arrays apply the super nested arrays [8]. More concretely, the difference coarray of the transmit array whose positions set 
$\mathbb{T}$ meets a super nested arrays is a hole-free virtual ULA, and the corresponding maximum DOF is

$$
f_{M}= \begin{cases}\frac{M^{2}}{2}+M-1, & \text { if } M \text { is even, } \\ \frac{(M+1)^{2}}{2}-1, & \text { if } M \text { is odd. }\end{cases}
$$

$$
\begin{aligned}
\mathbb{A} & =\left\{m_{j 1} d+n_{i 1} D d-m_{j 2} d-n_{i 2} D d \mid m_{j 1}, m_{j 2} \in \mathbb{T}, \quad j 1, j 2=1, \ldots, M ; n_{i 1}, n_{i 2} \in \mathbb{R}, i 1, i 2=1, \ldots, N\right\} \\
& =\left\{\left(m_{j 1}-m_{j 2}\right) d+\left(n_{i 1}-n_{i 2}\right) D d \mid m_{j 1}, m_{j 2} \in \mathbb{T}, \quad j 1, j 2=1, \ldots, M ; n_{i 1}, n_{i 2} \in \mathbb{R}, i 1, i 2=1, \ldots, N\right\},
\end{aligned}
$$

where $\left(m_{j 1}-m_{j 2}\right) d$ and $\left(n_{i 1}-n_{i 2}\right) d$ are the difference coarray of the transmit and receive arrays, respectively. Notice that $\mathbb{A}$ is a filled ULA if $D=f_{M}$. It is generally known that the super nested arrays have closed form expressions for the physical and virtual sensor locations for any given sensors number, so the proposed MIMO array also possesses the closed form sensor locations and free-holes DCSC.

To explain the structure of proposed MIMO array, it is convenient to generate the hole-free DCSC. Figure 1 provides a glance at the DCSC with parameters $M=N=8$. It can be observed that the one-side virtual aperture of DCSC becomes 760, where $D=f_{M}=39$.

Moreover, it is very clear that the difference coarray of transmit array with $M$ sensors is a filled ULA, and its DOF can be calculated by

$$
f_{M}=2 l_{M}+1
$$

where $l_{M}$ is the aperture length. In addition, let $D=f_{M}$, and then, the aperture length of DCSC can be written as

$$
L_{\mathrm{SC}}=l_{M}+l_{N} D=l_{M}+l_{N} f_{M} \text {, }
$$

As for the receive array, we assume that the sensor positions can be set as

$$
\left\{n_{i} D d \mid n_{i} \in \mathbb{R}, \quad i=1, \ldots, N\right\}
$$

where $\mathbb{R}$ means a location set satisfying the super nested arrays and $D$ is a positive integer. According to the definition of the DCSC in MIMO array, we can establish the DCSC set as

where $l_{N}$ means the aperture length of receive array with $N$ sensors. Hence, the DOF of DCSC is

$$
\begin{aligned}
f & =2 L_{\mathrm{SC}}+1=2 l_{M}+2 l_{N} f_{M}+1=f_{M}+2 l_{N} f_{M} \\
& =f_{M}\left(1+2 l_{N}\right)=f_{M} f_{N},
\end{aligned}
$$

where $f_{N}$ denotes the maximum DOF of receive array. Fortunately, we can find the optimal $M$ and $N$ to maximize $f$ for any given $K$. Firstly, we can gain the optimal of $M$ and $N$ under the fixed total number of sensors to maximize the DOF of transmit array in terms of the following equation:

$$
\begin{cases}M=N=\frac{K}{2}, & \text { if } K \text { is even, } \\ M=\frac{K+1}{2}, N=\frac{K-1}{2}, & \text { if } K \text { is odd. }\end{cases}
$$

Next, the optimal transmit and receive arrays' configuration is determined in the light of the property of super nested arrays. As a result, (14) can be recast as

$$
f=f_{M} f_{N}= \begin{cases}\left(\frac{M^{2}}{2}+M-1\right)\left(\frac{N^{2}}{2}+N-1\right), & \text { if } K \text { is even, } M \text { and } N \text { are both even, } \\ \left(\frac{M^{2}}{2}+M-\frac{1}{2}\right)\left(\frac{N^{2}}{2}+N-\frac{1}{2}\right), & \text { if } K \text { is even, } M \text { and } N \text { are both odd, } \\ \left(\frac{M^{2}}{2}+M-1\right)\left(\frac{N^{2}}{2}+N-\frac{1}{2}\right), & \text { if } K \text { is odd, } M \text { is even, } N \text { is odd. }\end{cases}
$$




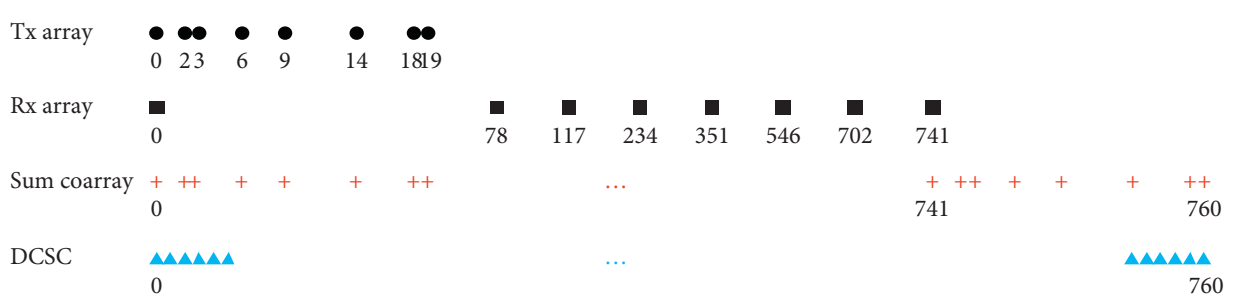

FIgURE 1: The structure of proposed MIMO array with $K=16$.

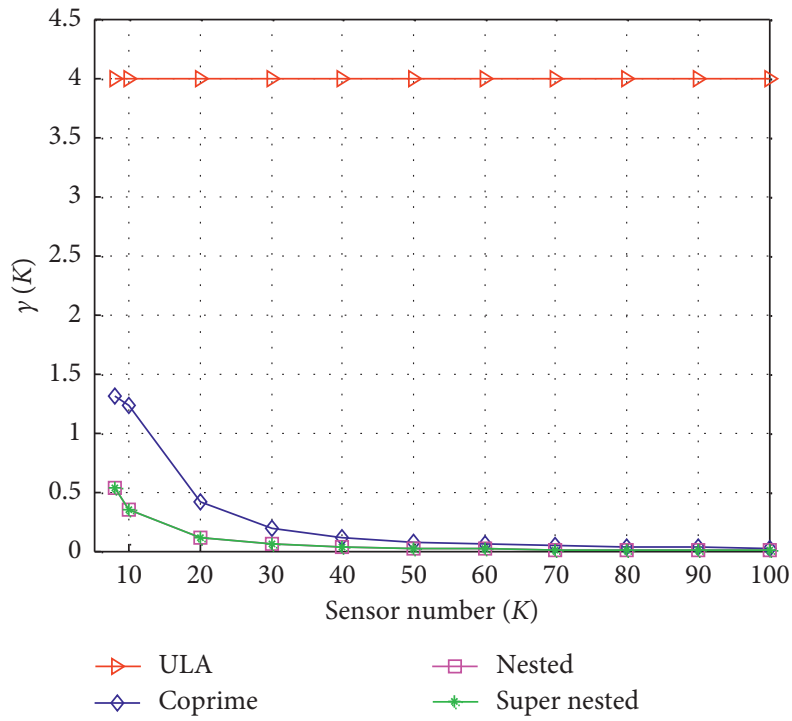

Figure 2: The DOF ratio with the sensor/pulse number $K$ varying from 8 to 100 .

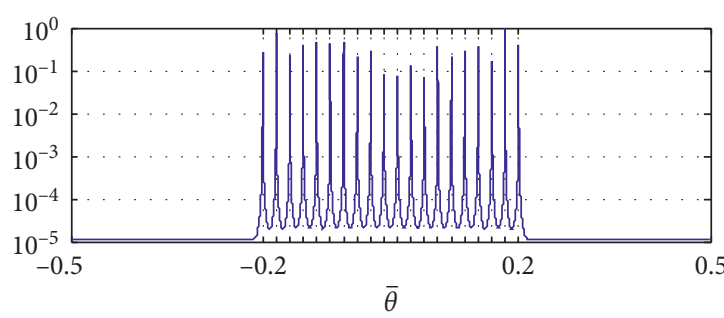

(a)

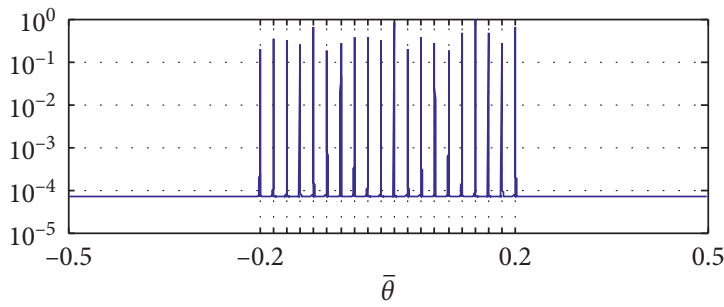

(c)

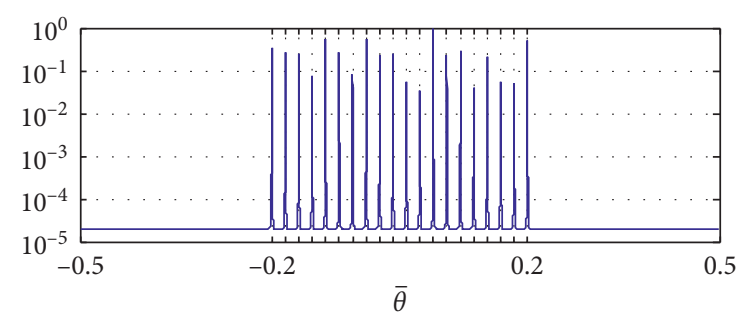

(b)

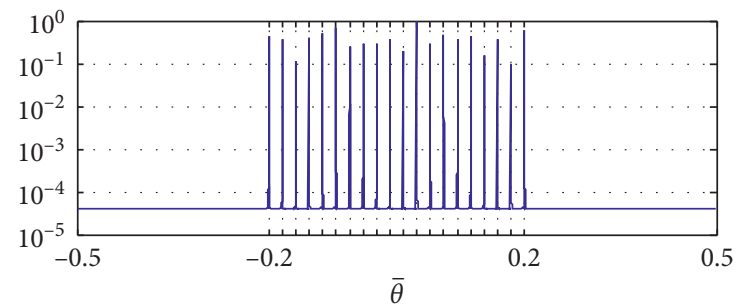

(d)

Figure 3: The MUSIC spectra $P(\bar{\theta})$ of four different methods without mutual coupling. (a) The MUSIC spectra $P(\bar{\theta})$ of ULA. (b) The MUSIC spectra $P(\bar{\theta})$ of coprime arrays. (c) The MUSIC spectra $P(\bar{\theta})$ of nested arrays. (d) The MUSIC spectra $P(\bar{\theta})$ of super nested arrays. 


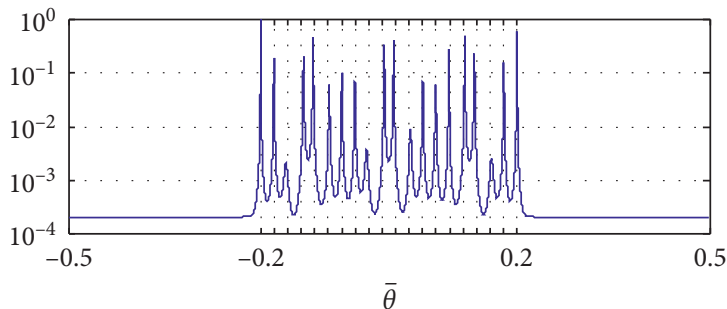

(a)

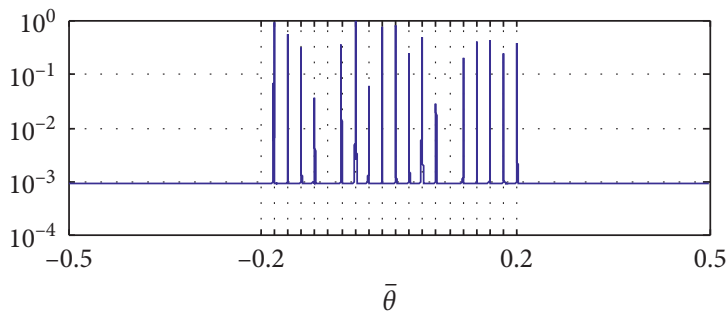

(c)

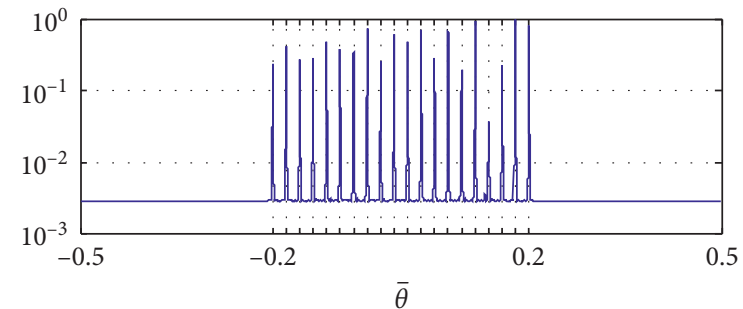

(b)

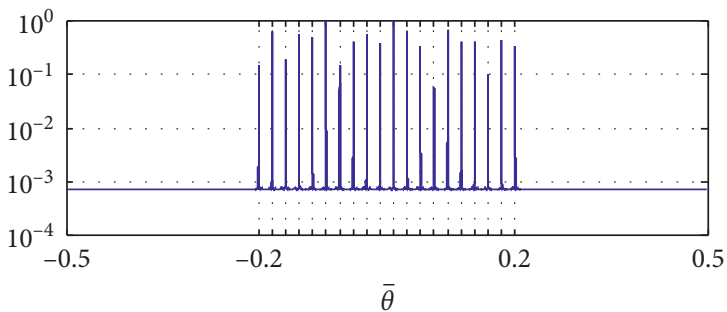

(d)

FIgURE 4: The MUSIC spectra $P(\bar{\theta})$ of four different methods in presence of mutual coupling. (a) The MUSIC spectra $P(\bar{\theta})$ of ULA. (b) The MUSIC spectra $P(\bar{\theta})$ of coprime arrays. (c) The MUSIC spectra $P(\bar{\theta})$ of nested arrays. (d) The MUSIC spectra $P(\bar{\theta})$ of super nested arrays.

It is easy to see that the proposed array structure can make use of $K$ physical sensors to simply obtain an optimal MIMO array which possesses larger DOF and less mutual coupling.

\section{Simulation Results}

In this section, we compare the performance of four kinds of array configurations: ULA [1], nested arrays, coprime arrays, and super nested arrays in the presence of mutual coupling. In our examples, we consider that the total number of sensors in MIMO array is $K=16$. For coprime arrays, we set $M=N=8$ with $M_{1}=N_{1}=2$ and $M_{2}=N_{2}=5$. The sensor locations of the transmit and receive arrays in super nested arrays are given by, respectively,

$$
\begin{aligned}
& \mathbb{A}_{T}=\{0,2,3,6,9,14,18,19\}, \\
& \mathbb{A}_{R}=\{0,78,117,234,351,546,702,741\} .
\end{aligned}
$$

To explain the algorithm overall performance appraisal, we will focus on DOF and MUSIC spectrum of DCSC.

3.1. Degrees of Freedom. Firstly, we demonstrate the capability of various array configurations to improve DOF in given sensors number $K$. Here, we define the DOF ratio as [24]

$$
\gamma(K)=\frac{K^{2}}{L(K)},
$$

where $L(K)=2 L+1$ represents the maximum continuous aperture length of DCSC. The smaller the $\gamma(K)$, the higher the DOF. Figure 2 reveals $\gamma(K)$ of four methods by varying $K$ from 8 to 100 . Sparse arrays such as coprime arrays, nested arrays, and super nested arrays have higher DOF than ULA. In particular, nested arrays and super nested arrays structures have higher DOF than the other two array structures in small $K$ and $\gamma(K)$ are close to 0 .

3.2. MUSIC Spectra. Finally, the MUSIC spectra of various array configurations are studied under the condition 500 samples and $0 \mathrm{~dB}$ SNR. In the examples, we set the mutual coupling parameters as $c_{1}=0.3 e^{j \pi / 3}, c_{l}=c_{1} e^{-j(l-1)(\pi / 8)} / l$ $(l=2, \ldots, B)$, and $B=100$. This is tested by assuming 20 sources lay in $\bar{\theta}_{i}=-0.2+0.4(i-1) / 19, i=1,2, \ldots, 20$, respectively. Figure 3 illustrates the MUSIC spectra $P(\bar{\theta})$ without mutual coupling, and $P(\bar{\theta})$ in Figure 4 is evaluated directly from the echo signal without using any decoupling methods in the presence of mutual coupling. If mutual coupling is negligible, all array configurations are capable of distinguishing 20 sources, as shown in Figure 3. Moreover, the performance of nested and super nested arrays shows the best, followed by coprime arrays, and then ULA for a given number of sensors due to the different DOF. However, nested arrays and ULA lose the ability to distinguish 20 sources because of the mutual couple effect. This is because the coupling weight coefficients of super nested arrays and coprime arrays are lower than that of nested arrays and ULA. In conclusion, the DCSC of the super nested MIMO array not only has the same array aperture as that of the nested MIMO array but also has large array spacing to lead to less mutual coupling.

\section{Conclusion}

In this paper, we study the problem of MIMO array with the higher DOF and less mutual coupling under any given sensors number. A novel sparse MIMO array whose transmit and receive arrays employ the super nested arrays is proposed. Firstly, the transmit and receive arrays are arranged as second-order super nested array, and the DOF 
of the difference coarray for the transmit array is $f_{M}$. Then, the interelement spacing of the receiving array is multiplied $f_{M}$ times in order to maximize the virtual array aperture. The condition in which the DCSC has no hole is determined. As a result, the closed form expression for sensor positions of the transmit and receive arrays are derived in any number of sensors. The simulation results of DOF and MUSIC spectra estimation prove the validity of the proposed MIMO array. Compared with coprime arrays and ULA structure, the proposed MIMO array can exhibit higher DOF in the same number of sensors. Compared with nested arrays and ULA structure, the proposed MIMO array can mitigate mutual coupling effect in the case of a fixed objective of DOF.

\section{Data Availability}

The datasets supporting the conclusions of this article are generated using MATLAB software by authors according to the radar parameters described in the manuscript.

\section{Conflicts of Interest}

The authors declare that they have no conflicts of interest.

\section{References}

[1] J. Li and P. Stoica, MIMO Radar Signal Processing, Wiley IEEE, Hoboken, NJ, USA, 2009.

[2] H. Ma, H. Tao, J. Su, and B. Liao, "DOD/DOA and polarization estimation in MIMO systems with spatially spread dipole quints," IEEE Communications Letters, vol. 24, no. 1, pp. 99-102, 2020.

[3] J. S. Kulkarni, R. Seenivasan, V. Abhaikumar, and D. R. P. Subburaj, "Design of a novel triple-band monopole antenna for WLAN/WiMAX MIMO applications in the laptop computer," Circuit World, vol. 45, no. 4, pp. 257-267, 2019.

[4] J. Kulkarni, A. Desai, and C.-Y. D. Sim, "Wideband four-port MIMO antenna array with high isolation for future wireless systems," International Journal of Electronics and Communications, vol. 128, pp. 1-14, 2021.

[5] E. Fishler, A. Haimovich, R. S. Blum, L. J. Cimini, D. Chizhik, and R. A. Valenzuela, "Spatial diversity in radars-models and detection performance," IEEE Transactions on Signal Processing, vol. 54, no. 3, pp. 823-838, 2006.

[6] J. Li and P. Stoica, "MIMO radar with colocated antennas," IEEE Signal Processing Magazine, vol. 24, no. 5, pp. 106-114, 2007.

[7] I. Bekkerman and J. Tabrikian, "Target detection and localization using MIMO radars and sonars," IEEE Transactions on Signal Processing, vol. 54, no. 10, pp. 3873-3883, 2006.

[8] C.-L. Liu and P. P. Vaidyanathan, "Super nested arrays: linear sparse arrays with reduced mutual coupling-part I: fundamentals," IEEE Transactions on Signal Processing, vol. 64, no. 15, pp. 3997-4012, 2016.

[9] W. Wang, L. Zou, and X. Wang, "A novel two-level nested STAP strategy for clutter suppression in airborne radar," Mathematical Problems in Engineering, vol. 2019, Article ID 2540858, 16 pages, 2019.

[10] M. Liu, X. Wang, and L. Zou, "Robust STAP with reduced mutual coupling and enhanced DOF based on super nested sampling structure," IEEE Access, vol. 7, pp. 175420-175428, 2019.
[11] P. P. Vaidyanathan and P. Pal, "Sparse sensing with co-prime samplers and arrays," IEEE Transactions on Signal Processing, vol. 59, no. 2, pp. 573-586, 2011.

[12] S. Qin, Y. D. Zhang, and M. G. Amin, "Generalized coprime array configurations for direction-of-arrival estimation," IEEE Transactions on Signal Processing, vol. 63, no. 6, pp. 13771390, 2015.

[13] J. Li, D. Jiang, and X. Zhang, "DOA estimation based on combined unitary ESPRIT for coprime MIMO radar," IEEE Communications Letters, vol. 21, no. 1, pp. 96-99, 2017.

[14] E. BouDaher, F. Ahmad, and M. G. Amin, "Sparsity-based direction finding of coherent and uncorrelated targets using active nonuniform arrays," IEEE Signal Processing Letters, vol. 22, no. 10, pp. 1628-1632, 2015.

[15] A. Raza, W. Liu, and Q. Shen, "Thinned coprime array for second-order difference co-array generation with reduced mutual coupling," IEEE Transactions on Signal Processing, vol. 67, no. 8, pp. 2052-2065, 2019.

[16] C. Zhou, Y. Gu, X. Fan, Z. Shi, G. Mao, and Y. D. Zhang, "Direction-of-arrival estimation for coprime array via virtual array interpolation," IEEE Transactions on Signal Processing, vol. 66, no. 22, pp. 5956-5971, 2018.

[17] M. Liu, B. Tang, X. Zheng et al., "STAP for airborne radar based on dual-frequency space-time coprime sampling structure," IEEE Access, vol. 8, pp. 138673-138681, 2020.

[18] C.-C. Weng and P. P. Vaidyanathan, "Nonuniform sparse array design for active sensing," in Proceedings of the 2011 Conference Record of the Forty Fifth Asilomar Conference on Signals, Systems and Computers (ASILOMAR), pp. 1062-1066, Pacific Grove, CA, USA, April 2011.

[19] M. D. Zoltowski, S. D. Silverstein, and C. P. Mathews, "Beamspace root-MUSIC for minimum redundancy linear arrays," IEEE Transactions on Signal Processing, vol. 41, no. 7, pp. 2502-2507, 1993.

[20] M. Liu, L. Zou, X. Yu et al., "STAP based on toeplitz covariance matrix reconstruction for airborne radar," Mathematical Problems in Engineering, vol. 2020, Article ID 6638425, , 2020.

[21] A. M. Elbir, "Direction finding in the presence of directiondependent mutual coupling," IEEE Antennas and Wireless Propagation Letters, vol. 16, pp. 1541-1544, 2017.

[22] W. Jeon, J. H. Kim, and S.-Y. Chung, "Effect of mutual coupling on uniform circular arrays with vector antenna elements," IEEE Antennas and Wireless Propagation Letters, vol. 16, pp. 1703-1706, 2017.

[23] J. Shi, G. Hu, X. Zhang, and F. Sun, "Sparsity-based DOA estimation of coherent and uncorrelated targets with flexible MIMO radar," IEEE Transactions on Vehicular Technology, vol. 68, no. 6, pp. 5835-5848, 2019.

[24] J. Liu, Y. Zhang, Y. Lu et al., "Augmented nested arrays with enhanced DOF and reduced mutual coupling," IEEE Transactions on Signal Processing, vol. 65, no. 21, pp. 5519-5563, 2017. 